\title{
COMUNICAÇÃO
}

\section{AVALIAÇÃO DO SULFATO DE AMINOSIDINE NO TRATAMENTO DA LEISHMANIOSE MUCOSA CAUSADA POR LEISHMANIA (VIANNIA) BRAZILIENSIS}

\author{
Gustavo A. Sierra Romero, Hélio A. Lessa, Vanize Macêdo, Edgar M. de Carvalho, Albino V. de \\ Magalhães, Maria de la Glória O. Orge, Maria V.A. de Abreu e Philip D. Marsden
}

O sulfato de aminosidine é uma droga com atividade leishmanicida comprovada ${ }^{6}$ que tem mostrado eficácia na leishmaniose visceral ${ }^{18}$ e no tratamento tópico ou sistêmico da forma cutânea da leishmaniose tegumentar americana (LTA) ${ }^{2} 3$. Realizamos um estudo prospectivo aberto que teve como objetivos avaliar a resposta dos pacientes com leishmaniose mucosa (LM) causada por Leishmania (Viannia) braziliensis, L(V)b, ao tratamento com aminosidine e a exequibilidade para sua aplicação em condições de trabalho em área endêmica. A pesquisa foi desenvolvida no sudeste do Estado da Bahia em área endêmica de LTA, onde existe transmissão quase exclusiva de L(V) $b^{4} 57$.

No período de setembro a novembro de 1994, foram incluídos no estudo, 21 pacientes com LM clinicamente ativa cujo diagnóstico baseou-se no exame clínico detalhado, no estudo histopatológico da biópsia da mucosa afetada, na inoculação do material de biópsia em hamsters, na intradermorreação de Montenegro (IDRM) e nos resultados da reação de imunofluorescência indireta (IFI) para anticorpos séricos antileishmânia. O diagnóstico foi comprovado parasitologicamente em $66,7 \%$ dos pacientes, sendo a inoculação de material da biópsia em hamsters, o método mais sensivel. A IDRM foi positiva em $100 \%$ dos pacientes e a IFI foi positiva em $66,7 \%$.

Os 21 pacientes foram submetidos ao tratamento com sulfato de aminosidine, $16 \mathrm{mg} / \mathrm{kg} /$ dia divididas em duas doses, pela via intramuscular, por 20 dias. O acompanhamento incluiu avaliações cada 10 dias durante o tratamento e posteriormente cada três mêses, durante um ano. O tempo médio de

Núcleo de Medicina Tropical, Universidade de Brasília, Brasília, DF, Laboratório de Imunologia do Hospital Universitário Prof. Edgard Santos, Universidade Federal da Bahia, Salvador, BA. Trabalho foi realizado com recursos do PCEDEN/MS e do NIH Grant Al- 30639.

Recebido para publicação em 16/01/96. acompanhamento foi de 12,6 meses. Realizaram-se estudos laboratoriais para determinar o aparecimento de toxicidade. Os pacientes que não conseguiram a remissão completa dos sinais e sintomas, três mêses depois de completar o esquema terapêutico, receberam tratamento alternativo com antimonial pentavalente.

A adesão ao tratamento foi de $100 \%$ e houve irregularidades na aplicação devido à falta de pessoal treinado para injetar a droga è dor no local da injeção. Os efeitos colaterais mais freqüentes foram dor no local da injeção e proteinúria leve.

Três mêses depois do tratamento, $13(61,9 \%)$ pacientes encontravam-se em remissão completa, 5 $(23,8 \%)$ apresentaram remissão parcial da doença, em $2(9,5 \%)$ houve falha terapêtica e $1(4,8 \%)$ paciente apresentou recidiva depois de ter alcançado a remissão completa.

Durante o período de acompanhamento de um ano, observou-se recidiva em $4(28,6 \%)$ de 14 pacientes e estavam clinicamente curados $10(47,6 \%)$ pacientes.

Com os resultados observados consideramos que o sulfato de aminosidine pode ser uma droga de segunda linha para o tratamento da LM.

\section{REFERÊNCIAS BIBLIOGRÁFICAS}

1. Chunge CN, Owate J, Pamba HO, Donno L. Treatment of visceral leishmaniasis in Kenya by aminosidine alone or combined with sodium sitibogluconate. Transactions of the Royal Society of Tropical Medicine and Hygiene $84: 221-225,1990$.

2. Correa Filho D. Estudo comparativo en1re antimoniato de meglumina, isetionato de pentamidina e sulfato de aminosidine, no tratamento de lesões cutâneas primárias causadas por Leishmania (Viannia) braziliensis. Tese de Mestrado. Universidade de Brasilia, Brasilia DF, 1993.

3. Krause G, Kroeger A. Topical treatment of American cutaneous leishmaniasis with paromomycin and methylbenzetonium chloride: a clinical study under field conditions in Ecuador. Transactions of the Royal Society 
Comunicação. Romero GAS, Lessa HA, Macêdo V, Carvalho EM, Magalhães AV, Orge MGO, Abreu MVA, Marsden PD. Avaliação do sulfato de aminosidine no tratamento da leishmaniose mucosa causada por Leishmania (Viannia) braziliensis. Revista da Sociedade Brasileira de Medicina Tropical 29:215-216, mar-abr, 1996.

of Tropical Medicine and Hygiene 88:92-94, 1994.

4. Marsden PD. Mucosal leishmaniasis due to Leishmania (Viannia) braziliensis L(V)b in Três Braços, Bahia-Brazil. Revista da Sociedade Brasileira de Medicina Tropical 27:93-101, 1994.

5. Marsden PD, Llanos-Cuentas EA, Lago EL, Cuba CC, Barreto AC, Costa JM, Jones TC. Human mucocutaneous leishmamiasis in Três Braços, Bahia-Brazil. An area of Leishmania braziliensis braziliensis transmission. III. Mucosal disease presentation and initial evolution. Revista da Sociedade Brasileira de Medicina Tropical 17:179-186, 1984.

6. Neal RA. The effect of antibiotics of the neomycin group on experimental cutaneous leishmaniasis. Annals of Tropical Medicine and Parasitology 62:54-62, 1968.

7. Rosa $\mathrm{AC}$, Cuba CC, Vexenat A, Barreto AC, Marsden PD. Predominance of Leishmania braziliensis in the regions of Três Braços and Corte de Pedra, Bahia, Brazil. Transactions of the Royal Society of Tropical Medicine and Hygiene 82:409-410, 1988.

8. Thakur CP, Bhowmick S, Dolfi L, Olliaro P. Aminosidine plus sodium stibogluconate for the treatment of Indian kala-azar: a randomaized dose-finding clinical trial Transactions of the Royal Society of Tropical Medicine and Hygiene 89:219-223, 1995. 